\title{
Articulating parametric resonance for an OWC spar buoy in regular and irregular waves
}

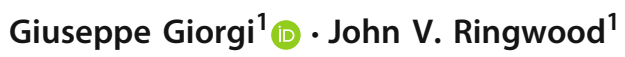

Received: 19 July 2018 / Accepted: 1 October 2018 / Published online: 10 October 2018

(c) Springer Nature Switzerland AG 2018

\begin{abstract}
Wave energy devices are designed, and controlled, to be extremely responsive to incoming wave excitation, hence, maximising power absorption. Due to the consequent large motion excursions, highly nonlinear behaviour is likely to occur, especially in relation to variations in wetted surface. Moreover, nonlinearities may induce parametric instability, or activate internal mechanisms for exchanging energy between different degrees of freedom (DoFs), usually affecting the overall efficiency of the device. Consequently, single-DoF linear models may produce overly optimistic power production predictions, and neglect important dynamics of the system. One highly nonlinear phenomenon, potentially detrimental to power absorption for several wave energy converters, is parametric roll/pitch; due to parametric excitation, part of the energy flow is internally diverted, from the axis where the power take-off is installed, to a secondary axis, generating parasitic motion. This paper proposes a computationally efficient multi-DoF nonlinear model, which can effectively describe nonlinear behaviour, such as parametric pitch and roll, and their impact on motion prediction, power production assessment, and optimal control parameters.
\end{abstract}

Keywords Parametric roll · Parametric resonance · Nonlinear Froude-Krylov force · Wave energy converters · Floating oscillating water column

\section{Introduction}

Exact and representative mathematical models are of paramount importance for effective design and optimization of wave energy converters (WECs). In the wave energy community, linear models are predominantly utilized, due to their simplicity and computational convenience, but often provide a poor description of the dynamical behaviour of the system. Indeed, conditions for linearity are seldom met, in wave energy applications, since the purpose of maximising power extraction requires motion exaggeration, consequently, enhancing nonlinear effects (Giorgi and Ringwood 2017b).

One remarkable example of an extremely nonlinear phenomenon, undetectable by linear models, is parametric resonance, which consists of an internal excitation mecha-

Giuseppe Giorgi

giuseppe.giorgi.2015@mumail.ie

John V. Ringwood

john.ringwood@nuim.ie

1 Centre for Ocean Energy Research, Maynooth University, Co. Kildare, Ireland nism, diverting a portion of the incoming energy away from the main degree of freedom, usually the one where wave energy is converted. Therefore, if not expressly exploited, parametric excitation is effectively a source of energy loss, eventually reducing the overall conversion efficiency. In addition, parametric resonance may also induce excessive loading on device components and moorings and increase operational costs. Consequently, the ability to model and predict such a phenomenon is valuable for both design and control purposes, so that parametric resonance can be prevented, or even taken advantage of Olvera et al. (2007). Furthermore, parametric motion should also be taken into account for survivability considerations, since excessive parametric motion may jeopardize the device integrity (Tarrant and Meskell 2016). Parametric resonance is traditionally described as a Mathieu type of instability (Fossen and Nijmeijer 2012), but, although it can give some insight on the conditions for occurrence of parametric roll, it cannot forecast its severity.

Since parametric resonance is due to time-varying system parameters (Fossen and Nijmeijer 2012), linear models, which only take the mean wetted surface into account, are inadequate. In contrast, it has been shown that parametric instability can be detected by nonlinear Froude-Krylov 
(FK) models, which integrate the static and dynamic pressure of the undisturbed wave field over the instantaneous wetted surface. Particular examples, in wave energy applications, are given by the SEAREV (Babarit et al. 2009) and the Wavebob (Tarrant and Meskell 2016) devices, for which parametric resonance is a detrimental parasitic effect, studied with a mesh-based nonlinear FK force model (LAMSWEC) (Gilloteaux 2007), which compares well with wave tank tests, provided an appropriate viscous drag description is included. Nonetheless, the main drawback of mesh-based nonlinear FK models is the computation time, since they require timeconsuming remeshing routines. For example, LAMSWEC, coded in Fortran, computes about ten times slower than real time (Gilloteaux 2007). Therefore, mesh-based nonlinear FK models are too slow to be extensively used for simulations, optimization, or control applications.

However, while mesh-based approaches are likely to be the only option for a geometry of arbitrary complexity, a computationally efficient method is available for axisymmetric devices (Giorgi and Ringwood 2017a). Note that the symmetry of revolution assumption is not particularly restrictive for point absorbers, since they are usually designed to be nondirectional, and are, therefore, axisymmetric. The nonlinear FK modelling approach has been widely validated in the literature, in its mesh-based implementation (LAMSWEC), see (Gilloteaux 2007; Babarit et al. 2009; Tarrant and Meskell 2016), among others. The analytical version of the nonlinear FK force calculation, used in this paper, has virtually identical accuracy of LAMSWEC, since it is just a faster implementation of the same equations, under the same hydrodynamic assumptions. Some validation of the model herein used is provided for a single degree-of-freedom (DoF) device, in Giorgi and Ringwood (2017b), and for a multi-DoF device, in Giorgi and Ringwood (2018a).

In this paper, an oscillating water column (OWC) spar buoy is considered, inspired by the Sparbuoy prototype device (Gomes et al. 2017), since wave tank tests have shown such a device to be particularly prone to parametric resonance. A 7 degree-of-freedom nonlinear model is defined (6 DoFs for the buoy plus one DoF for the heaving water column), which is fast enough to allow real-time computation. While a non-real-time model can be useful for simulation and power assessment, a fast nonlinear model, able to articulate parametric resonance, is required for design optimization, sensitivity analysis, or model-based control design, where many iterations may be required. In this paper, the sensitivity of parametric roll to the drag coefficient is studied, allowing designers to take more informed correcting measures to limit parametric roll.

Since parametric roll is a highly frequency-dependent phenomenon, both regular and irregular wave conditions are considered. Given the panchromatic nature of real sea states, long-duration simulations are needed to describe paramet-

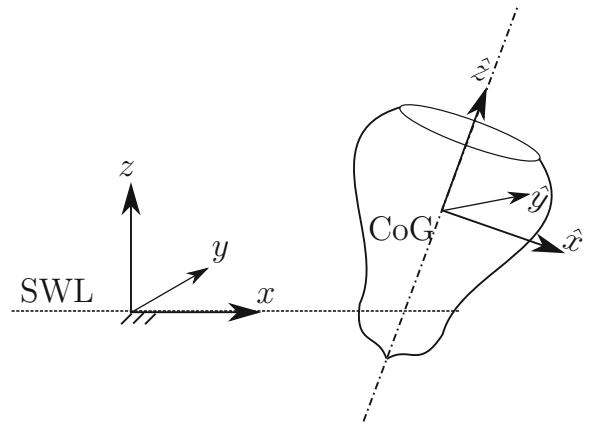

Fig. 1 Inertial frame $(x, y, z)$, with the origin at still water level (SWL), and non-inertial frame $(\hat{x}, \hat{y}, \hat{z})$, fixed with the body, with the origin at the centre of gravity of the body. The wave propagates in the positive $x$-direction.

ric resonance, as shown in (Malvar Ferreira 2016). However, with irregular waves, and according to the formulation of nonlinear FK forces in (Giorgi and Ringwood 2017a), the computational effort is directly proportional to the length of the simulation time. A major novelty of this paper, with respect to (Giorgi and Ringwood 2018b), is the reorganisation of the model, so that the computational burden is made insensitive to the simulated time window.

The remainder of the paper is organized as follows: Sect. 2 presents the nonlinear mathematical model, while Sect. 3 describes the device configuration and parameters. Some results are presented in Sect. 4, while Sect. 5 gives concluding remarks and considerations.

\section{Mathematical model}

Two right-handed frames of reference are introduced, as schematically shown in Fig. 1: an inertial frame $(x, y, z)$, with the origin at the still water level (SWL), $x$ pointing in the direction of propagation of the wave, and $z$ pointing upwards; a non-inertial frame $(\hat{x}, \hat{y}, \hat{z})$, fixed with the body, with the origin at the centre of gravity of the body. Assuming the fluid to be inviscid, and the flow to be irrotational and incompressible, linear potential theory can be formulated, defining the equation of motion for a generic single body, in the body-fixed frame of reference, as follows:

$M \ddot{\mathbf{x}}=\mathbf{f}_{F K_{\mathrm{st}}}+\mathbf{f}_{F K_{d y}}+\mathbf{f}_{d}+\mathbf{f}_{\mathrm{rad}}+\mathbf{f}_{\mathrm{vis}}+\mathbf{f}_{\mathrm{moor}}+\mathbf{f}_{\mathrm{PTO}}$,

where $M$ is the inertial matrix, $\mathbf{x}=(\hat{x}, \hat{y}, \hat{z}, \phi, \theta, \psi)$ is the state vector in the body-fixed frame, $\mathbf{f}$ are the generalized force vectors, composed of three forces $(\mathbf{F})$, and 3 torques (T). The force components on the right-hand side of (1) are the static and dynamic FK forces $\mathbf{f}_{F K_{s t}}$ and $\mathbf{f}_{F K_{d y}}$, respectively, the diffraction force $\mathbf{f}_{d}$, the radiation force $\mathbf{f}_{\text {rad }}$, the 
viscous force $\mathbf{f}_{v i s}$, the mooring force $\mathbf{f}_{\text {moor }}$, and the power take-off (PTO) force $\mathbf{f}_{P T O}$.

The mooring system is potentially an important factor for the modulation of parametric instability (Davidson 2017). Likewise, the viscous drag force may be essential in hydrodynamic models containing nonlinear FK force representation, to avoid unrealistic magnification of the motion, when parametric instability appears (Babarit et al. 2009). In this paper, linear radiation and diffraction forces are considered, which is a reasonable approximation for devices much smaller than the characteristic wave length (Falnes 2002). A computationally convenient state space representation has been used to model radiation forces, based on a moment-matching technique (Faedo et al. 2018).

Froude-Krylov forces correspond to the integral of the pressure of the undisturbed incident wave field, over the wetted surface of the device. Such a pressure is defined, according to linear Airy's theory, as follows:

$p(x, z, t)=p_{s t}+p_{d y}=-\gamma z+\gamma \frac{\cosh (k(z+h))}{\cosh (k h)} \eta(x, t)$,

where $p_{s t}=-\gamma z$ is the static pressure, $p_{d y}$ the dynamic pressure, $\gamma$ the specific weight of sea water, $\eta(x, t)$ is the free surface elevation of a two-dimensional wave, with amplitude $a=H_{w} / 2$ and wave period $T_{w}, k$ the wave number, and $h$ the water depth. It is also convenient to apply Wheeler stretching to (2), as shown in Giorgi and Ringwood (2018c).

Froude-Krylov forces $\left(\mathbf{F}_{F K}\right)$ and torques $\left(\mathbf{T}_{F K}\right)$ are computed by integrating the pressure, shown in (2), over the instantaneous wetted surface $S(t)$ :

$$
\begin{aligned}
\mathbf{F}_{F K_{s t}}+\mathbf{F}_{F K_{d y}}= & \mathbf{F}_{g}+\iint_{S(t)} p_{s t} \mathbf{n d} S+\iint_{S(t)} p_{d y} \mathbf{n} \mathrm{d} S \\
\mathbf{T}_{F K_{s t}}+\mathbf{T}_{F K_{d y}}= & \mathbf{r} \times \mathbf{F}_{g}+\iint_{S(t)} p_{s t} \mathbf{r} \times \mathbf{n} \mathrm{d} S \\
& +\iint_{S(t)} p_{d y} \mathbf{r} \times \mathbf{n} \mathrm{d} S
\end{aligned}
$$

where $\mathbf{F}_{g}$ is the gravity force, $\mathbf{n}=\left(n_{x}, n_{y}, n_{z}\right)$ is the unit vector normal to the surface, pointing outwards, $\mathbf{r}$ is the position vector, and $\times$ is the cross product.

Under linear approximation, a constant wetted surface is considered, in integrals ( $3 a$ ) and ( $3 b$ ), relying on the assumption of small relative motion between the wave and the device. In contrast, nonlinear FK forces are computed with respect to the instantaneous wetted surface, therefore, taking the real position of the device, with respect to $\eta$, into account.

For a geometry of arbitrary complexity, the calculation of the nonlinear FK integrals requires the use of plane pan- els to discretize the surface, which, consequently, has to be remeshed, at every time step, to define the instantaneous wetted surface (Gilloteaux 2007). Such a remeshing routine makes the approach computationally expensive. However, for axisymmetric buoys, a convenient parameterization of the wetted surface can ease the calculation of the FK integrals. Such a method, described hereafter, is validated in Giorgi and Ringwood (2018a).

The assumption of an axisymmetric geometry allows an analytical description of the complete wetted surface, therefore, avoiding computationally expensive mesh-based numerical techniques. The geometry of a generic buoy, symmetric around a vertical axis, can be conveniently described in cylindrical coordinates, with respect to the body frame $(\hat{x}, \hat{y}, \hat{z})$, as follows:

$$
\left\{\begin{array}{l}
\hat{x}(\varrho, \vartheta)=f(\varrho) \cos \vartheta \\
\hat{y}(\varrho, \vartheta)=f(\varrho) \sin \vartheta, \quad \theta \in[-\pi, \pi) \wedge \varrho \in\left[\varrho_{1}, \varrho_{2}\right], \\
\hat{z}(\varrho, \vartheta)=\varrho
\end{array}\right.
$$

where $f(\vartheta)$ is a generic function of the vertical coordinate $\varrho$, describing the profile of revolution of the axisymmetric body, as shown in Giorgi and Ringwood (2017b).

The change of coordinates, from Cartesian $(\hat{x}, \hat{y}, \hat{z})$ to cylindrical $(\varrho, \vartheta)$, shown in (4), requires the inclusion of $\left\|\mathbf{e}_{\varrho} \times \mathbf{e}_{\vartheta}\right\|$ in the integral, where $\mathbf{e}_{\varrho}$ and $\mathbf{e}_{\vartheta}$ are unit vectors in the $\varrho$ and $\vartheta$ directions, respectively. Furthermore, $\mathbf{n}$ can be expressed as $\frac{\mathbf{e}_{Q} \times \mathbf{e}_{\vartheta}}{\left\|\mathbf{e}_{Q} \times \mathbf{e}_{\vartheta}\right\|}$. Finally, since the integrals are defined in the body frame, it is necessary to map the pressure from the inertial frame (where it is defined) onto the body surface. The transformation, from $(x, y, z)$ to $(\hat{x}, \hat{y}, \hat{z})$, is represented by the Euler angle triad $(\phi, \theta, \psi)$, corresponding to roll, pitch, and yaw angles, respectively. The 3-2-1 Euler angle sequence is the rotation convention commonly used for marine vehicles, thought of as three sets of rigid rotations (Fossen 2011). To apply a rotation around the origin of the body frame (instead of around the origin of the inertial frame), a translation needs to be applied before and after the rotation, so that the two origins overlap at the moment of the rotation. Consequently, the integral for $\mathbf{F}_{F K_{d y}}$ in (3a), for example, becomes:

$\mathbf{F}_{F K_{d y}}=\iint_{S(t)} p_{d y}(\hat{x}, \hat{y}, \hat{z}) \mathbf{n} \mathrm{d} S=\int_{\vartheta_{1}}^{\vartheta_{2}} \int_{\varrho_{1}}^{\vartheta_{2}} p_{d y}(\varrho, \vartheta) \mathbf{e}_{\varrho} \times \mathbf{e}_{\vartheta} \mathrm{d} \varrho \mathrm{d} \vartheta$.

Although such an approach is applicable to any geometry with a symmetry of revolution, the vast majority of axisymmetric point absorbers can be described as a combination of 
cylinders, cones, and spheres. Note that discs (lids), which close the surface of a cylinder, cannot be described using cylindrical coordinates. To this end, polar coordinates are valid alternatives to cylindrical, as shown in Giorgi and Ringwood (2018a).

Finally, the FK force integrals must be solved numerically using, for example, a trapezoidal rule. The computation time depends on the integration scheme utilized, a 2D-quadrature scheme (Shampine 2008), and on the relative and absolute tolerances used to approximate the integral, both set to $10^{-3}$. The ultimate value of the computation time depends on hardware capabilities (Intel(R) Xeon(R) CPU (E5-1620 v3 @ $3.50 \mathrm{GHz}$ ) processor, with 16.0 GB RAM and Windows 7 Professional 64 bit), on the complexity of the geometry, and on the number of bodies and degrees of freedom considered. For the device studied in this paper, described in Sect. 3, the calculation time for a single update of the nonlinear FK forces, with regular waves, is between $1 \cdot 10^{-2} \mathrm{~s}$ and $4 \cdot 10^{-2} s$. The consequent run time for computing the response of the device depends on the discrete time solver scheme, the time step, and the simulation duration. Using a constant time step second-order Runge-Kutta scheme, varying the time step from $0.005 \mathrm{~s}$ to $0.025 \mathrm{~s}$, for a regular wave of period about $0.7 \mathrm{~s}$, the resulting run time is between one and three times the simulation time for a Matlab implementation. Therefore, such a method has the potential to run roughly in real time, or a little slower, depending on the particular implementation, given that Matlab is between one and two orders of magnitude slower than lower level coding languages, such as C or Fortran (Wendt et al. 2017). With a $\mathrm{C}$ or Fortran implementation, therefore, real-time execution is easily achievable. Nevertheless, although the mesh-based LAMSWEC nonlinear FK model is coded in Fortran (which is a significantly faster implementation than Matlab), it has a run time about ten times longer than the simulation time (Gilloteaux 2007); therefore, about one order of magnitude slower than the method used in this paper.

\section{Sparbuoy model}

The device studied in this paper is inspired by the Sparbuoy prototype WEC (Gomes et al. 2017), which is a floating OWC, extracting energy from the relative motion between a hollow spar buoy and the water column contained within. A 1:100 scale prototype is considered, for which wave tank experiments have highlight parametric resonance (Gomes et al. 2017). The shape and dimensions of the floater are shown in Fig. 2, while Table 1 tabulates relevant physical quantities $\left(z_{g}\right.$ the center of gravity, $z_{b}$ the center of buoyancy, $m$ the mass of the floater, $I$ the roll/pitch inertia, and $T_{n}$ the natural period). The water depth is $0.5 \mathrm{~m}$, equal to the depth of the wave tank for the experimental tests in Gomes

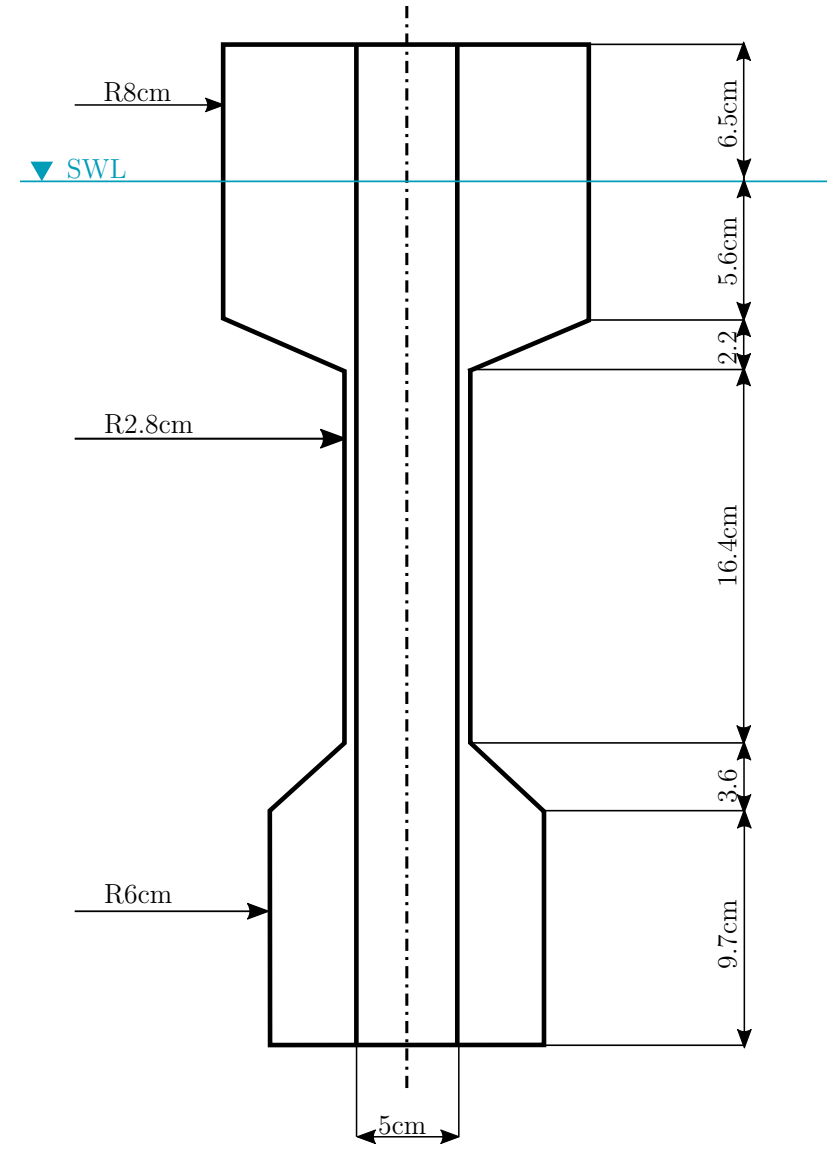

Fig. 2 Shape and dimensions of the Sparbuoy-like device, at 1:100 prototype scale

Table 1 Physical properties of the Sparbuoy-like device, at 1:100 prototype scale

\begin{tabular}{|c|c|c|c|c|c|}
\hline & & & $\begin{array}{l}\text { Surge } \\
\text { Sway }\end{array}$ & Heave & $\begin{array}{l}\text { Roll } \\
\text { Pitch }\end{array}$ \\
\hline$z_{g}$ & (m) & -0.205 & & & \\
\hline$z_{b}$ & (m) & -0.168 & & & \\
\hline$m$ & $(\mathrm{~kg})$ & 2.395 & & & \\
\hline$I$ & $\left(\mathrm{~kg} / \mathrm{m}^{2}\right)$ & 0.00733 & & & \\
\hline$T_{n}$ & (s) & & 12.7 & 0.89 & 1.35 \\
\hline
\end{tabular}

et al. (2017). Moorings are modelled, in surge and sway, as linear restoring coefficients, chosen to match the surge natural period, given in Gomes et al. (2017).

Mooring forces are known to potentially play an important role in parametric resonance generation, as discussed in Davidson (2017). However, since the purpose of the present paper is to show the ability of the nonlinear FK model to articulate hydrodynamically induced parametric roll, the mooring model has been extremely simplified. 
The Sparbuoy device can be modelled as a two-body system, considering the floater in 6 DoFs, with an additional heaving DoF for the water column, effectively modelled as a weightless rigid piston (Henriques et al. 2016). The hydrodynamic coefficients are computed with the boundary element method (BEM) software WAMIT (WAMIT Inc MIT 2014), which solves for the linear potential problem on the mean wetted surface of the device. Furthermore, WAMIT uses generalized modes for the computation of the moonpool-free surface (WAMIT Inc MIT 2014).

A linear turbine is considered, for which the ratio between the pressure difference in the chamber and the flow rate is constant $\left(k_{P T O}\right)$. Ignoring air compressibility effects, it follows that the force interchanged between the water column and the floater is (Sheng et al. 2015):

$F_{P T O}= \pm k_{P T O} A_{\mathrm{wc}}^{2}(\dot{\mathbf{x}}(3)-\dot{\mathbf{x}}(7))$,

where $A_{\mathrm{wc}}$ is the cross-sectional area of the water column; $\dot{\mathbf{x}}$ is the heave velocity of the floater $(\dot{\mathbf{x}}(3))$ and the water column $(\dot{\mathbf{x}}(7))$, respectively. The symbol \pm means that $F_{P T O}$ acts on the two bodies with opposite sign. The turbine coefficient $k_{P T O}$ is optimized for each wave condition, using the linear model, to maximise power capture (Sheng et al. 2015).

With the aim of defining a parsimonious model, nonlinear computation of FK forces are applied only to the floater's heave, roll, and pitch DoFs, since surge and sway is likely to be of little additional value in terms of accuracy, compared to the other DoFs for a point absorber (Giorgi and Ringwood 2018a). Nonlinear heave and pitch calculations, and their implementation, can be validated against WAMIT results (for modest device motion), as shown in Fig. 3, computing, under linear conditions, heave and pitch excitation force coefficients, $\overline{\mathbf{f}}_{\mathrm{ex}}$ (3) and $\overline{\mathbf{f}}_{\mathrm{ex}}(5)$, respectively. In fact, since WAMIT is a linear hydrodynamic code, it assumes very small motion and wave amplitude; therefore, the nonlinear model is run under linear conditions: floater kept in place, and extremely small incoming wave $\left(H_{w}=10^{-6}\right.$ $\mathrm{m}$ at 1:100 scale). Under such conditions, nonlinearities are negligible and linear and nonlinear results agree, validating the nonlinear code at one specific operating point. Likewise, the hydrostatic restoring forces, obtained with the WAMIT simulation, have been effectively compared with the nonlinear force/torque after an infinitesimal displacement, in the complete absence of waves.

\section{Results}

The response of the device is studied in both monochromatic waves, in Sect. 4.1, and panchromatic waves, in Sect. 4.2. In particular, a practical implementation issue, related to long
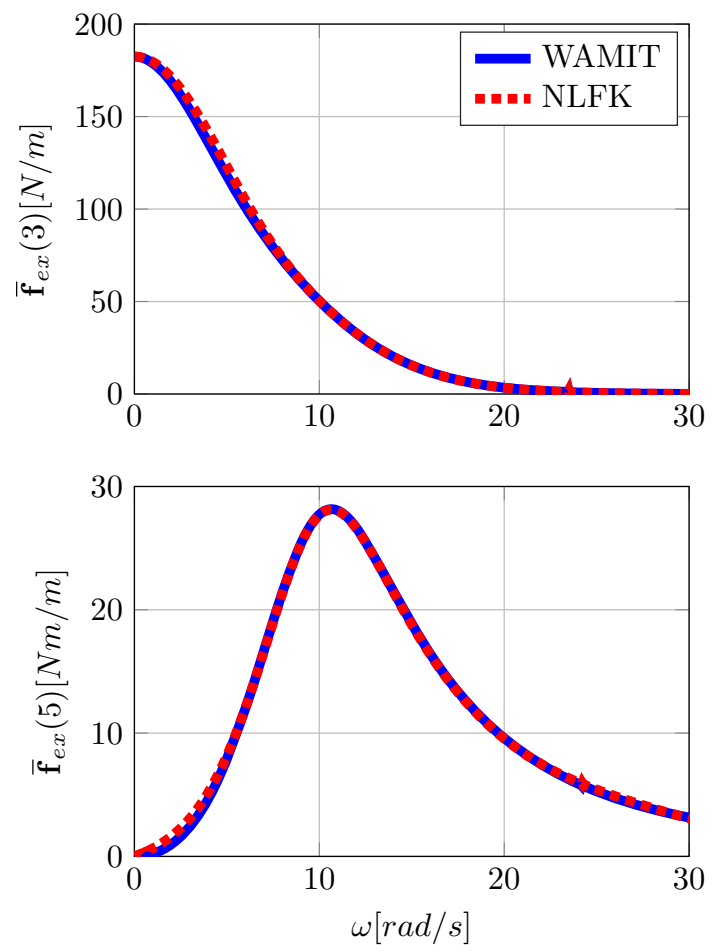

Fig. 3 Validation, against WAMIT results and in linear conditions $\left(H_{w}=10^{-6} \mathrm{~m}\right)$, of nonlinear Froude-Krylov (NLFK) calculations of excitation coefficients in heave and pitch

simulations with irregular wave conditions, is discussed and solved in Sect. 4.2.

\subsection{Monochromatic wave results}

Parametric instability appears when two conditions are satisfied: the amplitude of the excitation is larger than the damping of the secondary DoF, and the frequency of external excitation is about twice or equal to the natural frequency of the parametrically excited DoF. In fact, parametric roll can be described as a Mathieu type of instability, which is a single-DoF second-order differential equation, with non-dimensional damping coefficient $\mu$, and with harmonic variations (amplitude $a_{s}$, frequency $\omega_{s}$ ) of the stiffness term (Fossen and Nijmeijer 2012). It is possible to draw a stability diagram, shown in Fig. 4 , as a function of $\Delta$ and $\Lambda$, defined as follows:

$\Delta=\left(\frac{\omega_{n}}{\omega_{s}}\right)^{2}$
$\Lambda=a_{s}\left(\frac{\omega_{n}}{\omega_{s}}\right)^{2}$,

where $\omega_{n}$ is the roll natural period.

From Fig. 4, it can be seen that the instability regions are located around $\Delta$ equal to 0.25 and 1 , corresponding 


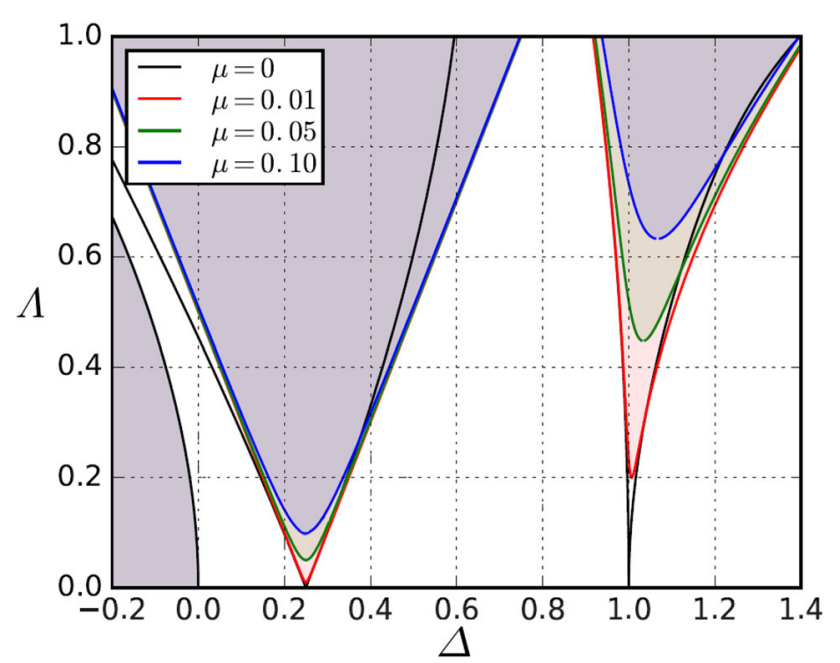

Fig. 4 Mathieu stability diagram for different non-dimensional damping coefficient values. The shaded areas represent the instability regions. Figure modified from Gomes et al. (2017)

to $\omega_{s}=\omega_{n}$ or $\omega_{s}=2 \omega_{n}$. On the other hand, the system becomes unstable only when the amplitude of the excitation is greater than the damping of the system.

Therefore, to study the amplitude and the frequency conditions, separately, simulations have been performed using a dense grid of wave conditions, with 11 wave heights, equally spaced between 0.005 and $0.0150 \mathrm{~m}$, and 76 wave periods, equally spaced between 0.5 and $2 \mathrm{~s}$. Note that such wave conditions are consistent with the 1:100 scale of the model considered, and consistent with experiments in Gomes et al. (2017).

Dissipation mechanisms, in the roll DoF, are the radiation damping, and viscous drag losses (which are modelled as an additional linear loss, proportional to velocity). Due to the lack of information available to determine an accurate viscous drag coefficient $\left(C_{D}\right)$, it is first set to zero (results from Figs. 5, 6, 7). However, to assess the influence of viscous effects, a sensitivity analysis on $C_{D}$ is also performed (results in Figs. 8, 9). Note that, in practice, to estimate the drag coefficient, fully nonlinear computational fluid dynamics (CFD) simulations or wave tank experiments are usually needed; Gomes et al. (2017), for example, study the logarithmic decrement of free decay tests, performed in a wave tank, to determine a linearized drag coefficient for the Sparbuoy model. The resulting maximum roll response, without drag, is shown in Fig. 5

As predicted by theory (Fossen and Nijmeijer 2012), and consistent with the experimental results (Gomes et al. 2017), parametric roll is localized at wave periods equal to, or half of, the roll natural period, while, elsewhere, the roll response is null. Likewise, it can be noted that parametric instability emerges after an amplitude threshold is passed, namely for a $0.009 \mathrm{~m}$ high wave, at $T_{w}=0.5 T_{n}$.

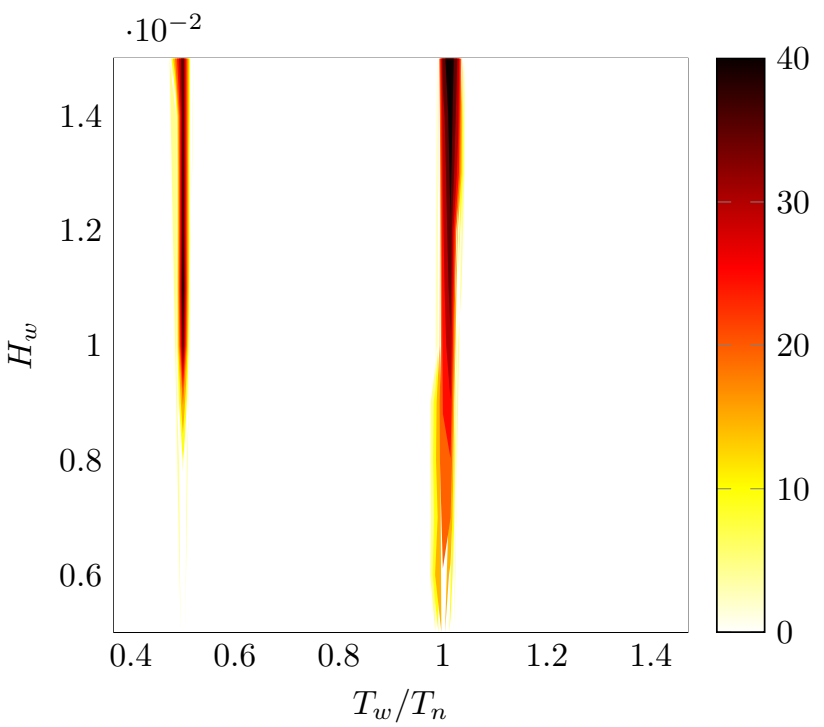

Fig. 5 Maximum roll response, in degrees, without viscous drag, for the SparBuoy-like device. Wave periods are normalized by the roll natural period

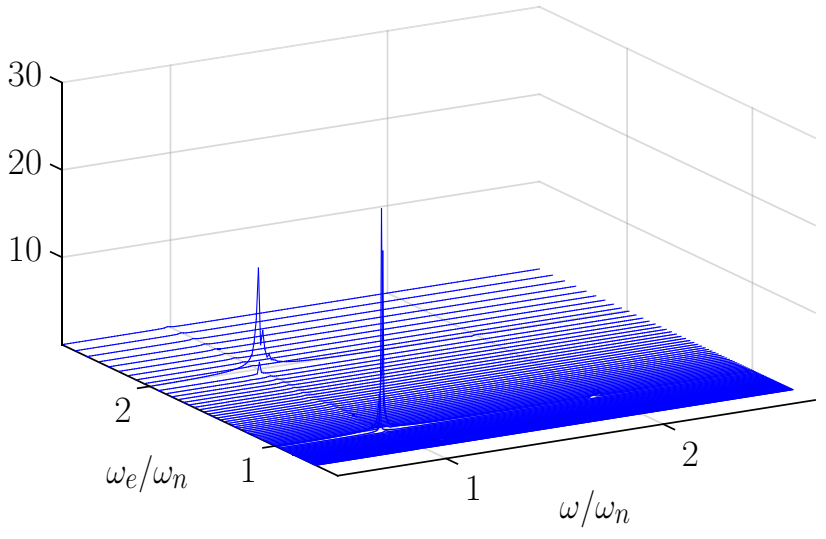

Fig. 6 Magnitude of the Fourier transform of the roll response, in degrees, at different excitation frequencies $\omega_{e}$, with wave amplitude of $0.01 \mathrm{~m}$ for the SparBuoy-like device. Both frequencies are normalized by the roll natural frequency $\left(\omega_{n}\right)$

Further insight can be gained by analysing the spectral energy content of the response of the device, both in roll and pitch. Considering, for the sake of argument, a representative wave height of $0.01 \mathrm{~m}$, the Fourier transform is computed for all wave periods, as shown in the waterfall plots in Figs. 6 and 7 , for roll and pitch, respectively. Both the Fourier frequency components $(\omega)$, and the excitation frequencies $\left(\omega_{e}\right)$, are normalized by the roll natural period. Note that results shown in Figs. 6 and 7 are consistent with similar results obtained for the WaveBob device, using the LAMSWEC mesh-based approach (Tarrant and Meskell 2016).

Considering Fig. 6, it is clear that the roll response is non-zero only for normalized excitation frequencies of 1 and 2 , and the spectrum has an evident peak at the normalized 


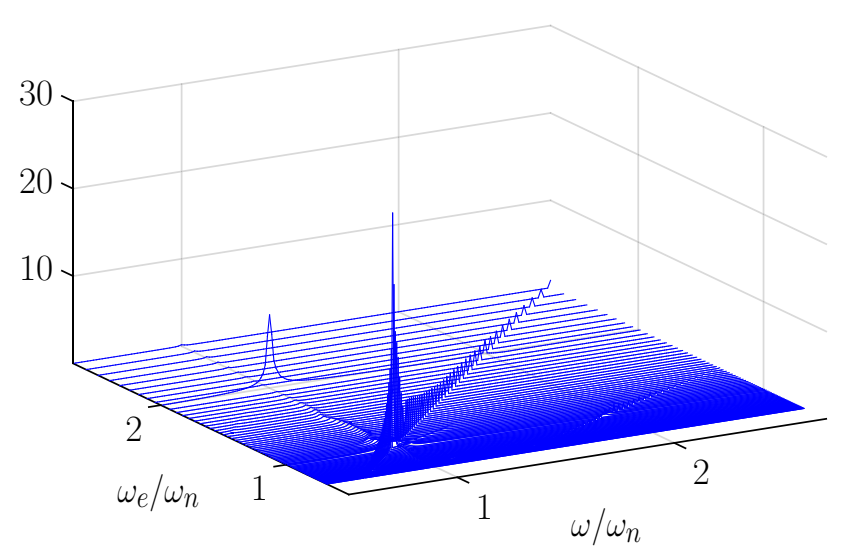

Fig. 7 Magnitude of the Fourier transforms of the pitch response, in degrees, at different excitation frequencies $\omega_{e}$, with wave amplitude of $0.01 \mathrm{~m}$ for the SparBuoy-like device. Both frequencies are normalized by the roll natural frequency $\left(\omega_{n}\right)$

frequency component of 1 . In fact, while the roll degree of freedom is never externally excited, parametric resonance activates an internal excitation mechanism at integer multiples of the normalized excitation frequency.

Conversely, since waves are externally exciting the device in the pitch DoF, the response spectrum shows a peak at the same excitation frequency; indeed, Fig. 7 shows that peaks align along the bisector of the horizontal plane (where $\omega=\omega_{e}$ ). Looking at the plot from a direction perpendicular to the direction of the bisector (direction $\omega=-\omega_{e}$ ), one can recognize the typical shape of the first-order pitch response. However, for a normalized excitation frequency of 2 , when parametric roll occurs, a peak in the pitch spectrum is generated at the pitch natural frequency.

Figures 5, 6, 7 demonstrate that the proposed modelling method is able to articulate parametric instability. However, though the likelihood of, and the conditions for, parametric instability generation are well described, the severity of the parametric response is overestimated. In fact, although the model considered in this study has simplified mooring and PTO systems, with respect to the one tested in Gomes et al. (2017), making the comparison less relevant, the maximum roll response obtained in the wave tank was about $21^{\circ}$, about half of that given by the model (about $40^{\circ}$, as shown in Fig. 5). Such a result is definitely consistent with the absence of viscous drag loss in the model for rotational DoFs. A similar issue is found, for example, in Babarit et al. (2009), where the LAMSWEC (mesh-based nonlinear FK) method is applied to the SEAREV device, without modelling viscous drag effects.

Therefore, to discuss the influence of viscous drag on the generation of parametric instability, a sensitivity study is performed. Considering a wave at half the pitch natural period (0.68 s), and $H_{w}=0.01 \mathrm{~m}, 11$ linear drag coefficients are tested, equally spaced between 0 and $0.01 \mathrm{Nms}$. The result-

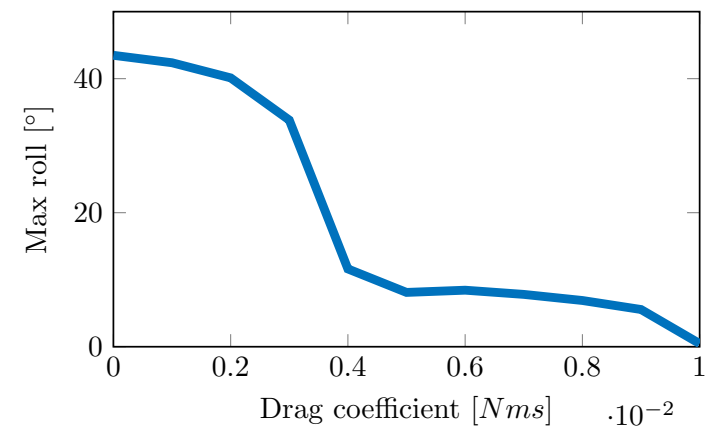

Fig. 8 Amplitudes of the steady-state roll responses, for a wave with $T_{w}=0.68 \mathrm{~s}$ and $H_{w}=0.01 \mathrm{~m}$, for 11 linear drag coefficients, equally spaced between 0 and $0.01 \mathrm{Nms}$

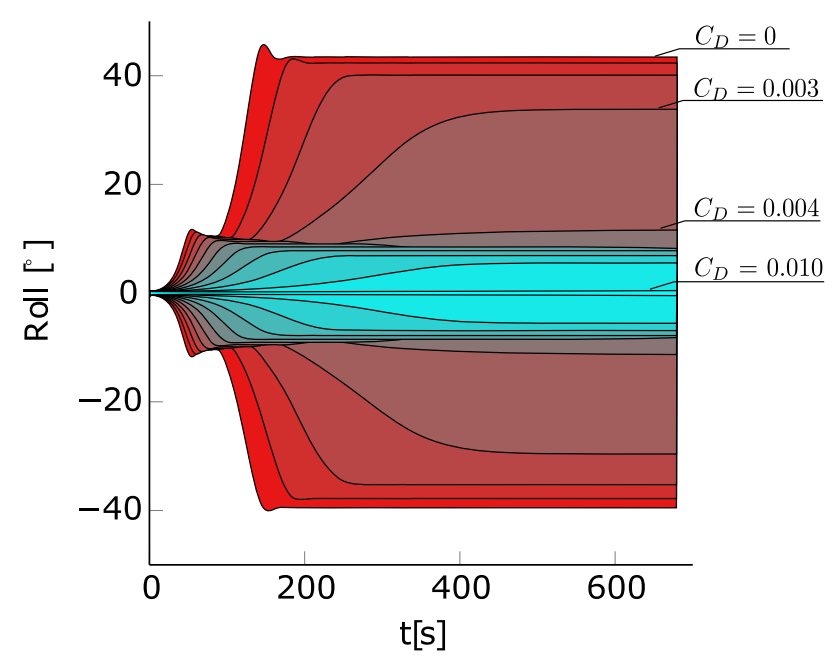

Fig. 9 For a wave with $T_{w}=0.68 \mathrm{~s}$ and $H_{w}=0.01 \mathrm{~m}$, for 11 linear drag coefficients, equally spaced between 0 and $0.01 \mathrm{Nms}$, each area corresponds to the envelop of the roll response time traces. As drag increases, roll response decreases

ing amplitudes of the steady-state responses are shown in Fig. 8, while the time traces are shown in Fig. 9, where each area represents the envelop of the time trace, using one drag coefficient.

As expected, larger linear drag coefficients cause the roll response to diminish, until it largely disappears for a drag coefficient of $0.01 \mathrm{Nms}$. In fact, one of the usual expedients adopted, to avoid parametric roll, is to increase viscous dissipation, for example through additional fins attached to the main floater body (Gomes et al. 2017). However, Fig. 8, which plots $1000 T_{w}$-long simulations, also shows that the transient time for building-up steady parametric roll significantly increases with the drag coefficient. Consequently, a very long transient, in regular wave conditions, would obviate the significance of parametric resonance in real sea conditions: given the panchromatic nature of irregular waves, there would be insufficient time to generate a sustained parametric response. Therefore, it is important to investigate the condi- 
tions for parametric resonance also in (realistic) irregular sea states, as will be carried out in Sect. 4.2.

\subsection{Panchromatic wave results}

From the discussion so far, it is clear that parametric resonance is an extremely frequency-dependent phenomenon. Regular waves steadily excite the device at the same frequency, facilitating the progressive build-up of parametric instability, when the excitation frequency is equal to, or twice, the resonant frequency in pitch/roll. However, real waves are panchromatic, so conditions for parametric excitation are weaker. In fact, comparing regular and irregular wave tank experiments, Malvar Ferreira (2016) shows that the roll response is more widely spread across the frequency range, but with lower maxima, although larger wave heights are used. Indeed, since parametric excitation is localized in a very narrow frequency window, a sustained parametric response is harder to achieve in the presence of a panchromatic wave process, as opposed to monochromatic excitation at the exact parametric resonant frequency.

Therefore, to verify the likelihood of a parametric response, with statistical reliability of the results, very long simulations/experiments are usually performed, to allow the device to escape the static equilibrium of a zero roll angle. As an example of 'very long', wave tank experiments for the SparBuoy device, in Malvar Ferreira (2016), last for about 1000 times the peak period, and a significant roll response is achieved only after $600 T_{p}$. While relatively long experimental tests do not usually represent a substantial issue, numerical simulations may be affected, as a consequence of a combination of the Nyquist sampling criterion [(imposing the bandwidth from the sampling rate (Basmann 1962)] and the condition for aperiodic realisation generation (imposing the frequency step from the realisation length (Mérigaud and Ringwood 2018): assuming a sampling frequency $F_{S}$ of $1.28 \mathrm{~Hz}$ (commonly implemented in wave data-buoys), the maximum frequency of the spectrum is $f_{\max }=F_{s} / 2$; on the other hand, a very long (aperiodic) time window (say $\left.T=1000 T_{p}\right)$ implies a tiny-frequency step $(\Delta f=1 / T)$ for correct realisation of the free surface elevation, since any panchromatic signal, regardless of the choice of random phases, is periodic, where the period is defined by the smallest frequency component (Mérigaud and Ringwood 2018). Therefore, using a small $\Delta f$ to reach $f_{\max }$ implies an excessive number of frequency components (up to 4000). Having so many frequency components is not a significant problem for linear models, since all forces are computed offline. On the contrary, time-domain nonlinear models are likely to compute nonlinear forces online, according to the actual relative position between the free surface elevation and the device. Nonlinear FK models are particularly adversely temporally affected by the number of spectral components, since

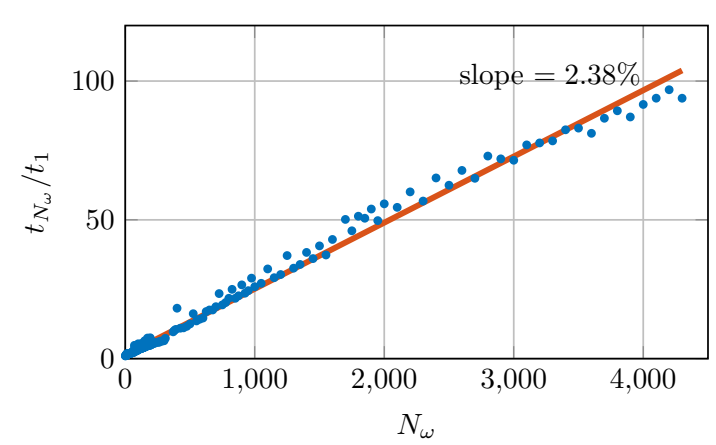

Fig. 10 Increase in computational time $t_{N_{\omega}}$, relative to the monochromatic limit $t_{1}$, for the numerical computation of nonlinear FroudeKrylov forces, with panchromatic waves, using $N_{\omega}$ spectral components

they have to consider the two-dimensional pressure field (both $x$ and $z$ dependence).

This is especially true for the 6-DoF numerical integration FK model which, while roughly achieving real-time computation with regular waves, becomes much slower when an excessive number of frequency components are included. A quantification of such a decline in computational performance is given in Fig. 10, which shows the increase in computational time for the calculation of FK forces, using $N_{\omega}$ components $\left(t_{N_{\omega}}\right)$, with respect to the regular wave case limit, where only one frequency component is used $\left(t_{1}\right)$. Starting from a $1000 T_{p}$-long realisation of an irregular sea spectrum, 500 equally spaced time samples of a $10 T_{p}$-long section are considered, to reduce the uncertainty regarding the computational time (which depends on the value of the integrals, with respect to relative and absolute tolerances, as well as the computing machinery). Consequently, equally spaced $N_{\omega}$ components are selected from within the bandwidth of the original process, with $N_{\omega} \in\left[1, N_{\omega}^{\max }\right]$, where $N_{\omega}^{\max }$ is the total number of components of the full process (4300). Note that $N_{\omega}=1$ correspond to a single frequency component, i.e., a regular wave.

Figure 10 shows an approximately linear increase in the relative computation time with the number of spectral components, with an interpolating line slope of $2.38 \%$. In particular, Fig. 10 proves that using all the components of the original spectrum represents an important practical issue, since the computation time may increase by up to a factor of 100. Such a significant slowdown would conflict with one of the main advantage of the nonlinear FK modelling approach in Giorgi and Ringwood (2017a), which is computational efficiency.

However, despite the fact that many frequency components are required to generate a statistically random long realisation (in time), the local (in space, at a given time) wave and pressure fields are likely to be much less complex; therefore, needless of such a refined discretization in 
the frequency domain. In fact, the numerical integration is performed, at each time step, using the wave and pressure fields in the (relatively small) spatial region where the device is sitting. Therefore, at every time instant, $t_{0}$, a computationally cheaper reduced model is sought (offline), which can efficiently and accurately describe the pressure field in the local body location, i.e., in the $x-z$ domain where the device is expected to operate.

Two approaches for the identification of the local pressure field are hereafter examined, namely a fitting approach and an FFT-based approach. According to the fitting approach, the parameters $\left(\alpha_{i}\right)$ of an alternative structure $(\widetilde{p})$ are chosen, so that the error with respect to the actual pressure field is minimized. The most convenient structure is found to be the following:

$$
\begin{aligned}
\widetilde{p}= & \alpha_{0} \\
& +\sum_{i=1}^{\widetilde{N_{\omega}}}\left[\alpha_{i} \cos \left(k_{i} x\right)+\alpha_{i+\widetilde{N_{\omega}}} \sin \left(k_{i} x\right)\right] \frac{\cosh k_{i}(z+d)}{\cosh k_{i} d},
\end{aligned}
$$

where $\widetilde{N_{\omega}}$ is the number of frequency components of the reduced representation, selected within the bandwidth of the original process, to obtain equally spaced levels of spectral energy; in this way, the spectrum is used to inform the definition of the fitting structure. The primary merit of the representation in (9) is that, while resembling the original pressure field representation, in (2), it is linear in the parameters, leading to a simple least-squares quadratic optimization problem, easy to solve analytically. Note that $\widetilde{N}_{\omega}$ is a variable defining both the accuracy of the fit, and the size of the reduced model, since the number of parameters is $2 \widetilde{N}_{\omega}+1$.

Accurate results are achievable, provided that the fitting grid size and density is properly chosen. However, such a choice would require a convergence study, and the evaluation of the model's accuracy outside the fitting grid. Since the second option (FFT-based) is found to be less arbitrary and less sensitive to the parameters choices, the fitting approach has been discarded.

According to the FFT-based approach, at each time step (say $t_{0}$ ) a portion of the time trace of $\eta$ is considered, over a time window centred on $t_{0}$; an FFT is then applied to this portion, to compute its frequency domain representation. Since the time window over which the FFT is computed is relatively short, compared to the overall simulation time, the frequency step (hence the number of frequency components) is small. Such frequency components are used, during the simulation, to reconstruct the free surface elevation $\widehat{\eta}$, and pressure field $\widehat{p}$, at $t=t_{0}$, and over a spatial domain local to the device. Since such components are used just at $t=t_{0}$, the non-periodicity at the extremes of the time window is irrelevant.

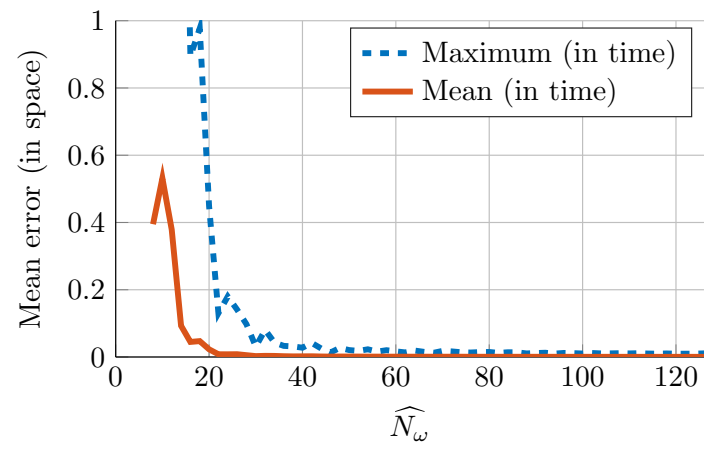

Fig. 11 Maximum and mean error, using the FFT-based approach for pressure representation reduction, with respect to the number of spectral components

However, since different wave components travel at different speed, the accuracy of the reconstructed field (in space) depends on the length of the time window, as well as the length of the spatial domain. Note that the sampling frequency and the length of the time window define the number of frequency components of the identified model. The length and depth of the considered spatial domain is roughly defined by the expected operational space spanned by the device.

On one hand, such an identification method implies a negligible overhead, thanks to efficient algorithms for FFT computation. On the other hand, a considerable speed up in computational time is achieved, since accurate results are easily obtained with less than 100 components (see Fig. 11), as opposed to several thousand.

To define the error index, shown in Fig. 11, consider a spatial domain $x \in[-0.15 \mathrm{~m}, 0.15 \mathrm{~m}]$ and $z \in[-0.20 \mathrm{~m}, \eta]$, selected according to the dimensions of the device, shown in Fig. 2; from a $1000 T_{p}$-long realisation of a JONSWAP spectrum with $T_{p}=0.68 \mathrm{~s}, H_{s}=0.02 \mathrm{~m}$, and $\gamma=3.3$, a portion is selected, taking 201 time samples, at the sampling frequency of $1.28 \mathrm{~Hz}$, centred at the highest peak of $\eta(100$ samples at each side), which are sufficient to give a significant description of the possible range of errors. At each time instant, the mean of the absolute value of the normalized relative error, between the identified $\widehat{p}$ and the original $p$, is computed over the spatial domain. Figure 11 shows the consequent maximum and average errors, in time, depending on the number of components $\widehat{N_{\omega}}$ of the reduced representation, showing that very accurate results can be achieved already with $\widehat{N_{\omega}}=50$. Note that, given the $2.38 \%$ increase rate of the computational time with $\widehat{N_{\omega}}$, shown in Fig. 10, $\widehat{N_{\omega}}=85$, for example, would just double the computational time, with respect to the computation for a regular wave, while producing a negligible error, as shown in Fig. 11. This compares favourably with a 100-fold increase in computation, in the absence of model reduction.

Finally, using the FFT-based reduction method, the response of the SparBuoy device is studied for a comprehen- 


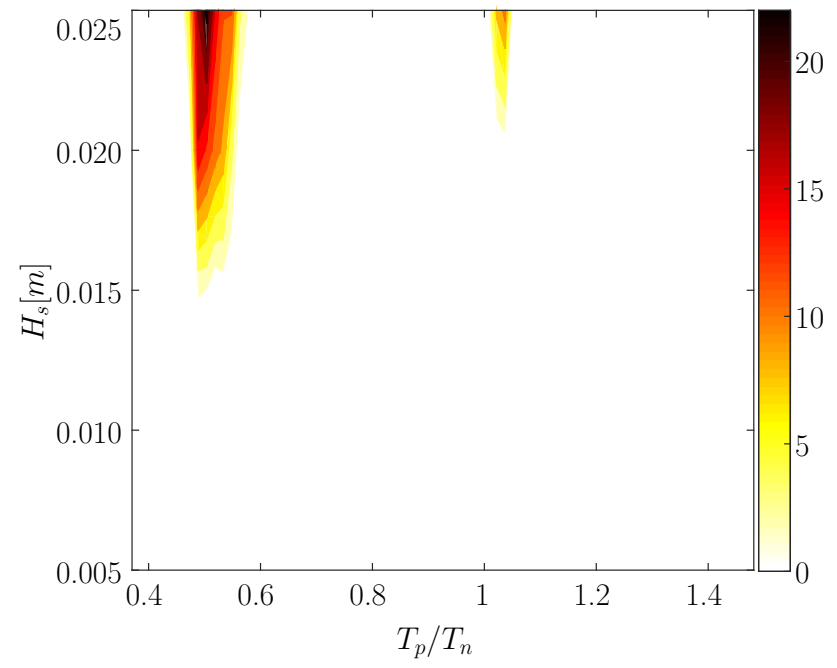

Fig. 12 Maximum roll response, in degrees, without viscous drag, in irregular wave conditions, for the SparBuoy-like device. Wave periods are normalized by the roll natural period

sive range of sea states, based on JONSWAP spectra, with $T_{p}$ ranging from 0.5 to $1.6 \mathrm{~s}$, with a $0.02 \mathrm{~s} \mathrm{step}$, and $H_{s}$ ranging from 0.005 to $0.025 \mathrm{~m}$, with a $0.005 \mathrm{~m}$ step, considering $500 T_{p}$-long realisations. Note that larger waves are considered, with respect to the regular wave case, since parametric excitation is weaker, due to the panchromatic nature of the incoming waves. This approach is consistent with the experimental tests in (Malvar Ferreira 2016), where maximum significant wave heights, with irregular waves, are larger than the maximum wave heights of the regular waves.

In Sect. 4.1, no viscous drag is initially included, so a sensitivity analysis, with regard to the viscous drag coefficient, is subsequently performed. Furthermore, Fig. 12 presents the maxima of the roll responses for all the considered sea states, comparable to Fig. 5.

As expected, although, with larger significant wave heights, the roll response with irregular waves is smaller than with regular waves. Furthermore, the frequency range where parametric resonance appears, but still centred at $T_{p} / T_{n}$ values of 0.5 and 1 , is wider, due to the panchromatic nature of the excitation. Comparing Fig. 12 with Fig. 5, it can be noted that the roll response is more significant at $T_{p} / T_{n}=0.5$ with irregular waves, while at $T_{p} / T_{n}=1$ with regular waves. Although no general explanation can be found (the Mathieu equation can model only the likelihood of instability, not its severity), it can be inferred that, since smooth nonlinearities tend to transfer spectral energy to frequencies which are integer multiples of the exciting frequencies, it is more likely for parametric resonance to be stronger at higher frequencies $\left(T_{p} / T_{n}=0.5\right)$.

Furthermore, note that a sustained parametric response typically requires a long transient, as shown in Fig. 9, for regular waves. In irregular wave conditions, such an extended

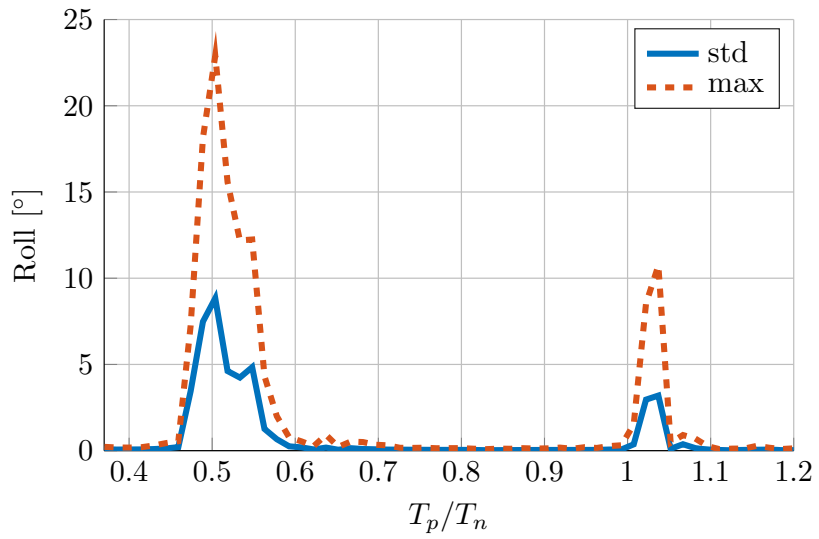

Fig. 13 Maximum (max) and standard deviation (std) of the roll response, without viscous drag, with $H_{s}=0.025 \mathrm{~m}$, for the SparBuoylike device. Wave periods are normalized by the roll natural period

transient is particularly unfavourable to the generation of a significant roll response, due to the panchromatic nature of the excitation and the frequency dependence of parametric instability. Therefore, it is reasonable to infer that parametric response is likely to be more severe at higher frequencies $\left(T_{p} / T_{n}=0.5\right)$, since faster transients can be expected.

Figure 12 only shows the maximum roll response, while it is interesting to also considered the standard deviation, to provide a more significant evaluation of the overall behaviour of the system. Figure 13 shows a cross section of the map in Fig. 12, at $H_{s}=0.025 \mathrm{~m}$. In the region where parametric roll is significant, the maximum response is between 2.5 and 5 times the standard deviation.

Finally, for Fig. 8, a sensitivity analysis is performed, shown in Fig. 14, to evaluate the degree to which viscous drag affects the eventual parametric excitation of the device, for the sea state of maximum parametric response $\left(T_{p}=0.68 \mathrm{~s}\right.$, $\left.H_{s}=0.025 m\right)$. Comparing Fig. 14 to Fig. 8, similar trends are found, but, although larger waves are used in irregular conditions, a smaller roll response is obtained. Such information has the potential to inform the design process, suggesting an ideal viscous drag coefficient in the roll DoF, achieved, for example, with lateral fins, as in Gomes et al. (2017), such that parametric roll is largely avoided.

\section{Conclusions}

Parametric resonance is a highly nonlinear phenomenon, often unexpected by wave energy converter designers. In fact, at an early design stage, when significant modifications to the device concept, operating principle, shape, and dimensions take place, overly-simplistic linear models are commonly used, due to their computational convenience. However, parametric resonance, undetectable by linear models, is usually 


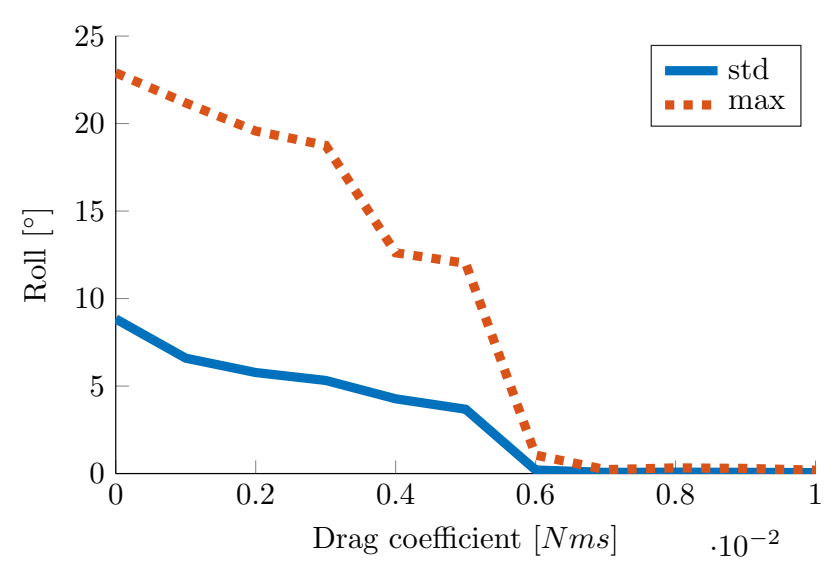

Fig. 14 Maximum (max) and standard deviation (std) of the roll response, depending on the viscous drag coefficient, with $T_{p}=0.68 \mathrm{~s}$ and $H_{s}=0.025 \mathrm{~m}$, for the SparBuoy-like device

discovered (with some dismay!) after the first-wave tank tests, at a stage where there is less design freedom, due to the financial investment already incurred. Therefore, since parametric roll is usually detrimental, suboptimal mitigating actions take place, to contain the undesired phenomenon.

On the contrary, having a computationally viable mathematical model, able to describe nonlinearities, including parametric resonance, is potentially beneficial for informing the design process of the real device dynamics, before prototype construction and experimentation. Furthermore, reliable knowledge of the device dynamics can hopefully channel device design into the exploiting of nonlinear behaviour, instead of mitigation. In particular, since parametric instability is an extreme magnification of a small perturbation, a dedicated design that takes advantage of parametric resonance is potentially highly beneficial for power production. A well-informed control strategy may exploit parametric resonance to generate the conditions for maximum power generation.

Such device/control design approaches require a fast-andrepresentative model of the device. The present paper takes a step in this direction, implementing a computationally convenient modelling approach for nonlinear Froude-Krylov force calculation for an axisymmetric wave energy converter, able to compute almost in real time, despite a relatively slow coding language, but already an order of magnitude faster than previous similar models. The ability of such a method to articulate parametric excitation is demonstrated by considering a Sparbuoy-like device, known to have a parametric response. Such a mathematical model can be useful for design investigations, for example exploring the sensitivity of parametric roll generation to viscous drag variations. Results show a significant dependence of parametric response on viscous losses, not only in the amplitude of the roll response, but also in the length of the transient required for a sustained response, in regular waves. Furthermore, since it is shown that parametric excitation is weaker in irregular waves, it is convenient to focus design considerations (for example designing the dimension of lateral fins, which increase viscous drag in the rotational degree of freedom) in irregular wave conditions, to avoid over-conservative design.

Acknowledgements This paper is based upon work supported by Science Foundation Ireland under Grant No. 13/IA/1886.

\section{References}

Babarit A, Mouslim H, Clément AH, Laporte-Weywada P (2009) On the numerical modelling of the nonlinear behaviour of a wave energy converter. In: Proceedings of the ASME 2009 28th international conference on ocean, offshore and arctic engineering, Honolulu

Basmann R (1962) Probability and statistics: the Harald Cramer volume. JSTOR, Leicester

Davidson J, Ringwood JV (2017) Mathematical modelling of mooring systems for wave energy converters-a review. Energies https:// doi.org/10.3390/en10050666

Faedo N, Peña-Sanchez Y, Ringwood JV (2018) Finite-order hydrodynamic model determination for wave energy applications using moment-matching. Ocean Eng 163:251-263

Falnes J (2002) Ocean waves and oscillating systems. Cambridge University Press, Cambridge

Fossen TI (2011) Handbook of marine craft hydrodynamics and motion control. Wiley, Amsterdam

Fossen TI, Nijmeijer H (2012) Parametric resonance in dynamical systems. Springer, New York

Gilloteaux JC (2007) Mouvements de grande amplitude d'un corps flottant en fluide parfait. Application á la recuperation de l'energie des vagues. $\mathrm{PhD}$ thesis, Ecole Centrale de Nantes-ECN

Giorgi G, Ringwood JV (2017a) Computationally efficient nonlinear Froude-Krylov force calculations for heaving axisymmetric wave energy point absorbers. J Ocean Eng Mar Energy 3(1):21-33

Giorgi G, Ringwood JV (2017b) Froude-Krylov and viscous drag representations in nonlinear wave energy devices models in the computation/fidelity continuum. Ocean Eng 141:164-175

Giorgi G, Ringwood JV (2018) Analytical representation of nonlinear Froude-Krylov forces for 3-DoF point absorbing wave energy devices. Ocean Eng 164(2018):749-759

Giorgi G, Ringwood JV (2018b) Parametric motion detection for an oscillating water column spar buoy. In: Proceedings of the 3rd international conference on renewable energies offshore RENEW, Lisbon

Giorgi G, Ringwood JV (2018c) Relevance of pressure field accuracy for nonlinear Froude-Krylov force calculations for wave energy devices. J Ocean Eng Mar Energy 4(1):57-71. https://doi.org/10. 1007/s40722-017-0107-5

Gomes RPF, Malvar Ferreira JDC, Riberio de Silva S, Henriques JC, Gato LMC (2017) An experimental study on the reduction of the dynamic instability in the oscillating water column spar buoy. In: Proceedings of the 12th European wave and tidal energy conference, Cork

Henriques JC, Portillo JC, Gato LMC, Gomes RPF, Ferreira DN, Falcão AF (2016) Design of oscillating-water-column wave energy converters with an application to self-powered sensor buoys. Energy 112:852-867. https://doi.org/10.1016/j.energy.2016.06.054

Malvar Ferreira JDC (2016) Experimental study of the dynamic instability in the oscillating water column spar buoy. July, Instituto Superior Tecnico, University of Lisbon, Tech. Rep 
Mérigaud A, Ringwood JV (2018) Free-surface time-series generation for wave energy applications. IEEE J Ocean Eng 43(1):19-35. https://doi.org/10.1109/JOE.2017.2691199

Olvera A, Prado E, Czitrom S (2007) Parametric resonance in an oscillating water column. J Eng Math 57(1):1-21. https://doi.org/10. 1007/s10665-006-9048-z

Shampine LF (2008) Matlab program for quadrature in 2D. Appl Math Comput 202(1):266-274. https://doi.org/10.1016/j.amc.2008.02. 012

Sheng W, Alcorn R, Lewis A (2015) Hydrodynamics of OWC wave energy converters. Renew Energy Offshore pp 489-496. https:// doi.org/10.1201/b18973-68. http://www.crenetbase.com/doi/10. 1201/b18973-68

Tarrant KR, Meskell C (2016) Investigation on parametrically excited motions of point absorbers in regular waves. Ocean Eng 111:6781. https://doi.org/10.1016/j.oceaneng.2015.10.041
WAMIT Inc., MIT (2014) User manual Wamit. http://www.wamit.com/ manualv7.2/wamit_v72manual.html

Wendt FF, Yu YH, Nielsen K, Ruehl K, Bunnik T, Touzon I, Nam BW, Kim JS, Kim KH, Janson CE (2017) International energy agency ocean energy systems task 10 wave energy converter modeling verification and validation. In: Proceedings of the 12th European wave and tidal energy conference, technical committee of the European wave and tidal energy conference, Cork

Publisher's Note Springer Nature remains neutral with regard to jurisdictional claims in published maps and institutional affiliations. 